\title{
Difference between breast milk jaundice and breast-feeding jaundice: literature review
}

\author{
Rabie Abdul Hakim Shihab ${ }^{1 *}$, Ahmed Mohammad Bin Samman', Fotoun Abubaker Aqeel', \\ Ziyad Tawfik Ghabrah ${ }^{4}$, Omar Tawfik Ghabrah ${ }^{4}$, Abdullah Nizar Ghannam ${ }^{4}$, \\ Abdulrahman Hassan Alasmari ${ }^{5}$, Hessa Abdulkareem Abahussain ${ }^{6}$, \\ Abdulaziz Abdulrhman Alsulami ${ }^{7}$, Samia Mhros Alamri ${ }^{8}$, Rawan Abdulrahman Babader', \\ Doaa Ahmed Aljehani ${ }^{9}$
}

\author{
${ }^{1}$ Department of Pediatric Intensive Care Unit, Al Aziziyah Children Hospital, Jeddah, Saudi Arabia \\ ${ }^{2}$ College of Medicine, Umm Al-Qura University, Mecca, Saudi Arabia \\ ${ }^{3}$ Department of Pediatrics, East Jeddah Hospital, Jeddah, Saudi Arabia \\ ${ }^{4}$ Department of Pediatrics, Maternity and Children Hospital, Al Kharj, Saudi Arabia \\ ${ }^{5}$ Department of Orthopaedics, Khamis Mushait General Hospital, Khamis Mushait, Saudi Arabia \\ ${ }^{6}$ College of Medicine, Almaarefa University, Riyadh, Saudi Arabia \\ ${ }^{7}$ Department of Emergency Medicine, King Abdullah Hospital, Jeddah, Saudi Arabia \\ ${ }^{8}$ Department of Pediatrics, Maternity and Children Hospital, Medina, Saudi Arabia \\ ${ }^{9}$ College of Medicine, Ibn Sina National College, Jeddah, Saudi Arabia
}

Received: 06 April 2021

Accepted: 17 April 2021

*Correspondence:

Rabie Abdul Hakim Shihab,

E-mail: Rshihab@moh.gov.sa

Copyright: (C) the author(s), publisher and licensee Medip Academy. This is an open-access article distributed under the terms of the Creative Commons Attribution Non-Commercial License, which permits unrestricted non-commercial use, distribution, and reproduction in any medium, provided the original work is properly cited.

\begin{abstract}
Evidence shows that the different etiologies of neonatal jaundice, including breastfeeding and breast milk jaundice, have many different aspects. Therefore, the present study aims to conduct a literature review to compare breastfeeding and breast milk jaundice, ehich will furtherly help physicians and healthcare practitioners to have adequate information to properly establish an accurate diagnosis. The precise cause of breast milk jaundice is unclear. The majority of the suggested etiologies include factors found in human breast milk. Other theories point to possible genetic defects in the infected neonates. It has been reported that pregnane-3a,20ß-diol, epidermal growth factor, interleukin (IL) $1 ß$, alphafetoprotein, and B-glucuronidase are several factors that are solid constituents of the breast milk which may attribute to the development of this jaundice. Reports showed that breast milk jaundice usually develops in 20-30\% of U.S. neonates, and most of whom are breastfed. Moreover, studies showed that more than one-third of infants on breastfeeding will eventually have high serum bilirubin levels that are $\geq 5 \mathrm{mg} / \mathrm{dl}$. Evidence showed that the diagnosis should be considered when the levels of serum bilirubin exceed $5 \mathrm{mg} / \mathrm{dl}$. Breast milk jaundice will usually fade away with no interventions, and in some cases, phototherapy inauguration and breast milk discontinuation might be needed. In breastfeeding jaundice, serum bilirubin levels usually peak within the first five or six days of life due to wrong or inadequate breastfeeding practices, and the management should be done by correcting these habits, while phototherapy might be applied when the bilirubin levels exceed $18-20 \mathrm{mg} / \mathrm{dl}$.
\end{abstract}

Keywords: Breast milk, Breastfeeding, Jaundice, Bilirubinemia 


\section{INTRODUCTION}

Jaundice or hyperbilirubinemia is frequently reported as a clinical condition in neonates. Evidence from previous research even indicated that hyperbilirubinemia will eventually affect around two-thirds of neonates, owing to physiological or pathological conditions. ${ }^{1}$ Ullah et al previously suggested that a diagnosis of hyperbilirubinemia or jaundice should be established when the total serum bilirubin (TSB) reaches, within the first six days after delivery, a level that is above $95^{\text {th }}$ percentile for age or when the levels are considered high risk for developing complications and clinical picture on the affected neonates. Studies showed that although bilirubin is an essential element in the antioxidant mechanisms of the neonates, elevated serum billirubin levels can cause serious complications through hindering the development of the central nervous system and other complications. ${ }^{2-5} \mathrm{~A}$ diagnosis of jaundice is then established by the clinical presentation of having a yellowish discoloration of the skin and sclera, mainly, following the high rise in the TSB levels.

Previous investigations reported that infants on breastfeeding tend to develop hyperbilirubinemia than other infants that are fed on formula milk. ${ }^{6}$ It has been shown that breastfeeding and breast milk jaundice are the two common causes of this phenomenon. In 1963, Arias et al described the first case of neonatal unconjugated hyperbilirubinemia that lasted beyond the $3^{\text {rd }}$ week after delivery in infants that were breastfed. ${ }^{7}$ It is now wellknown that the development of neonatal jaundice usually occurs within the $1^{\text {st }}$ to $2^{\text {nd }}$ weeks of life, and does not usually last without any interference even without discontinuation of breastfeeding. ${ }^{6,8}$ However, persistence for up to 12 weeks has been recorded for some cases. On the other hand, breastfeeding jaundice insufficient or reduced caloric intake through breastfeeding during the first days of life. The pathology is usually with earlyonset. ${ }^{9}$ However, there are many aspects to be considered for differentiating between the two types to establish a proper diagnosis.

Evidence shows that the different etiologies of neonatal jaundice, including breastfeeding and breast milk jaundice, have many different aspects. For instance, hyperbilirubinemia trends in both etiologies differ, as neonates with breast milk jaundice tend to have very high peaks of serum bilirubin that tend to normalize slowly along the course of the disease pathology. Moreover, a differential diagnosis of the many etiologies of jaundice should be made before establishing the final diagnosis. ${ }^{10}$ Therefore, the present study aims to conduct a literature review to compare breastfeeding and breast milk jaundice, hoping that it will furtherly help physicians and healthcare practitioners to have adequate information to properly establish an accurate diagnosis.

\section{METHODS}

A systematic search was conducted to identify relevant studies in the following databases: pubmed, medline, web of science, embase, google scholar, and scopus. The following search terms were used ('breast milk jaundice' or 'breastmilk jaundice') and ('breast feeding jaundice' or 'breastfeeding jaundice'). The reference lists were manually searched to identify additional relevant studies meeting inclusion criteria. We included any study that reports the epidemiology, clinical characteristics, outcomes of management and differences between breast milk jaundice and breastfeeding jaundice. No restrictions were applied.

\section{DISCUSSION}

\section{Breast milk jaundice}

\section{Etiology}

The precise cause of breast milk jaundice is unclear. The majority of the suggested etiologies include factors found in human breast milk. Other theories point to possible genetic defects in the infected neonates. It has been reported that pregnane-3a,20ß-diol, epidermal growth factor, interleukin (IL)1ß, alpha-fetoprotein, and $\beta$ glucuronidase are several factors that are solid constituents of the breast milk which may attribute to the development of this jaundice. ${ }^{11}$ The first factor has been linked with decreased bilirubin disposal through the potential prevention of conjugation. Moreover, evidence showed that B-glucuronidase, which is a naturally occurring compound, also plays an important role in the development of breast milk jaundice by inducing the intestinal reabsorption of the bilirubin and decreasing the potential excretion. This mechanism is done through inhibition of the normal conjugation and deconjugation processes. ${ }^{6}$ It has also been reported among studies in the literature that the activities of this enzyme are essential in the breast milk of the mother while the same activities have been shown to be limited in the formula-made milk. ${ }^{12}$ IL $1 \beta$ and various amounts of epidermal growth factors have also been reported to be present within the breast milk of the mothers, which play a role in inducing cholestasis and production of hyperbilirubinemia. ${ }^{11}$

The explanation for this is that this substance increases bilirubin resorption and decreases intestinal motility in the neonatal cycle, resulting in higher unconjugated bilirubin levels. ${ }^{6}$ It has been found that the levels of alphafetoprotein are usually high in cases of breast milk jaundice. The mechanism behind this is still unclear. Many investigations have previously indicated the potential presence of defects within the area that is responsible for UGT1A1 genetic coding make it more likely to develop this type of jaundice. Moreover, it has been shown that if any mutations will occur in this region, Gilbert and Crigler-Najjar and syndromes will eventually develop, leading to secondary and chronic elevation in the serum 
bilirubin levels. ${ }^{13}$ Consequently, it has been indicated that any mutations within the UGT1A1 gene can subsequently induce a state of hyperbilirubinemia and jaundice. ${ }^{6}$ Moreover, previous trials have investigated the effect of breast milk administration on the potential mechanisms for induction of hyperbilirubinemia and if there are any roles on the enterohepatic circulation. ${ }^{14,15}$

\section{Epidemiology}

It has been estimated that breast milk jaundice usually develops in $20-30 \%$ of U.S. neonates, and most of whom are breastfed. ${ }^{16}$ Moreover, studies showed that more than one-third of infants on breastfeeding will eventually have high serum bilirubin levels that are $\geq 5 \mathrm{mg} / \mathrm{dL}$. Moreover, the levels are expected to be even higher within the first three weeks after delivery in $2-4 \%$ of these infants, with the bilirubin levels recording up to $10 \mathrm{mg} / \mathrm{dL} .^{1}$ A rate of $20-28 \%$ of infants in Turkey and Taiwan have also been reported to develop breast milk jaundice within the first weeks of life. ${ }^{13}$ However, no concise estimation for the global prevalence of breast milk jaundice has been found in the literature. On the other hand, previous reviews suggested that it is mostly equal to that of the United States. Moreover, we did not find any evidence that any sex could be possibly related to having a higher prevalence rate than the other. ${ }^{17}$

\section{Diagnosis and evaluation}

Breast milk jaundice usually appears during the first two weeks of life in an otherwise stable breastfed child. These babies gain weight normally and produce sufficient amounts of urination and defecation excreta. ${ }^{6}$ Regarding the appropriate levels at which a diagnosis of jaundice should be established, evidence showed that the diagnosis should be considered when the levels of serum bilirubin exceed $5 \mathrm{mg} / \mathrm{dL}$, however, observations for neonates that developed higher bilirubin levels than $1.5 \mathrm{mg} / \mathrm{dL}$ should be continuously updated. Following the laboratory assessment of the bilirubin levels, clinical observation of the color of the skin of the affected infants is the next step. It is now well-established that the yellowish discoloration will first appear on the face and sclera and might eventually extend to the other parts of the body including the trunk and extremities.

Before diagnosing breast milk jaundice, a patient with hyperbilirubinemia must undergo a workup to possibly identify the underlying etiology behind the elevated bilirubin levels. The attending physicians should assess the levels of both the total, direct and indirect bilirubin levels among the suspected patients. A diagnosis of direct hyperbilirubinemia or cholestasis should be established when the levels of the conjugated bilirubin within the serum of the affected patients rise to or more than $20 \%$ of the assessed bilirubin levels, which is usually equal to 1 $\mathrm{mg} / \mathrm{dL}$. In such cases, however, assessment of other underlying etiologies as hepatitis, sepsis, biliary atresia, and similar diseases affecting the biliary flow should be suspected. It should also be noted that the levels of the unconjugated bilirubin can also be elevated in patients with breast milk jaundice, and patients with hemolytic anemia, and therefore, a proper differential diagnosis should be conducted. ${ }^{17}$

The incompatibility of the blood groups of the neonates, the deficiency of the glucose-6-phosphate dehydrogenase, and other hemolytic diseases as familial spherocytosis can also lead to the development of severe hemolysis inducing a state of hyperbilirubinemia. ${ }^{17,18}$ Therefore, assessment for these diseases should also be considered by conducting related tests as hematocrit, hemoglobin values, coombs tests, genetics, and blood profiling. Moreover, other hyperbilirubinemia-related diseases might also involve Gilbert or Crigler-Najjar syndrome, and should be considered by the clinician but should not be examined until jaundice lasts longer than the average normal period for the pathology of the development of breast milk jaundice. Moreover, unconjugated hyperbilirubinemia might be caused by galactosemia and hypothyroidism, but these disorders should be ruled out. ${ }^{6,19}$

\section{Management}

The previous guidelines by the American Academy of Pediatrics phototherapy showed that management of infants with breast milk jaundice is not always necessary until the serum bilirubin levels exceed the maximum limits. ${ }^{20}$ At first, phototherapy should be inaugurated, and if the modality manages to reduce the serum bilirubin levels below $12 \mathrm{mg} / \mathrm{dL}$, breastfeeding is encouraged subsequently. Breastfeeding is furtherly encouraged even when the levels are higher than $12 \mathrm{mg} / \mathrm{dL}$ but are found to be less than the pre-specified levels by the American Academy of Pediatrics' phototherapy. $^{6}$ Moreover, discontinuing breastfeeding for one day might be helpful in cases when the serum bilirubin levels exceed 20 $\mathrm{mg} / \mathrm{dL} .{ }^{17}$

\section{Breastfeeding jaundice}

Reports have shown that bilirubin levels in infants that have had either artificial or breastfeeding were similar within the first five or six days after delivery. However, in patients who are breastfed, estimates showed that they have higher levels of serum bilirubin within physiologic jaundice. Although many clinicians have assumed that such elevations may be normal and physiologic, others have reported that they are not and pathological. ${ }^{21,22}$ It is worth-mentioning that calling breastfeeding jaundice this name might have caused such conflicts, and some authors prefer to refer to it as breast non-feeding jaundice to differentiate it from the breast milk type. Moreover, a definitive definition of breastfeeding jaundice has also been an area of conflict. This is because early studies did not discuss the mechanism of breastfeeding and the associated factors that may affect the development of the phenomenon. For instance, previous studies have demonstrated that the production of breast milk can be 
affected by the ingestion of water or other solution before the initiation of breastfeeding. ${ }^{23-27}$

Reduced milk production will adversely affect the health of the relevant baby leading to a state of deprivation and starvation. Besides, investigations reported that delayed meconium emptying or meconium aspiration might also be associated with elevated levels of serum bilirubin through stimulation of the intestinal absorption. ${ }^{28,29}$ On the other hand, a previous study reported that breastfeeding does not affect the process of bilirubin synthesis. ${ }^{30}$ Therefore, reduced breastfeeding and poor related nursing practices are the most possible causes for developing this type of jaundice. This can be supported by the relation between the weight of the affected babies and the bilirubin levels. ${ }^{31}$ Studies have shown that reduced bilirubin levels were associated with weight gain in the affected infants and vice versa. $^{32,33}$ Moreover, the higher the frequency of breastfeeding the less the estimated levels of serum bilirubin. ${ }^{34,35}$ Therefore, reduced frequencies of breastfeeding by harmful practices, as prior ingestion of water or other solutes, or by late lactation initiation, might affect the bilirubin levels, causing breastfeeding jaundice. ${ }^{36}$ Although the pathology might be limited to the first five days after delivery, the subsequent events might last secondary to reduced caloric intake, leading to the development of some complications as lethargy, dehydration, and severe weight loss. ${ }^{37}$

\section{Management of breastfeeding jaundice}

The management of breastfeeding jaundice can be simply done by correction of the breastfeeding habits and related problems that have caused the elevation in the serum bilirubin levels. Moreover, management by phototherapy and enhanced approaches of feeding might be suggested only when the serum levels of bilirubin are as high as 18$20 \mathrm{mg} / \mathrm{dL}$. Moreover, careful evaluation of other etiologies of hyperbilirubinemia should be approached and observations on adequate and appropriate breastfeeding should be done even with the slowly rising serum bilirubin levels. Generally, encouraging breastfeeding practice for mothers should be early approached within the first hours after delivery. Moreover, maintaining the adequate and frequent supply of breast milk with the normal practices is also necessary to avoid the development of serious hyperbilirubinemia that can cause encephalopathy.

\section{Association of breast milk and breastfeeding jaundice}

Although we have discussed that both types of jaundice differ in many aspects, evidence shows that each one of them might affect the other. It has been indicated that patients who had a history of elevated bilirubin levels within the first five days of life, owing to haemolysis, or breastfeeding jaundice, usually develop higher serum bilirubin concentrations within the following $2^{\text {nd }}$ and $3^{\text {rd }}$ weeks. This was justified by the potential effect of the initial elevation of bilirubin levels, which may have created a pool of bilirubin within the affected infants. Moreover, it has been shown that even with the enhancement of the enterohepatic circulation and the frequent ingestion of breast milk, bilirubin levels are still elevated. ${ }^{38}$

\section{CONCLUSION}

In the present review, we have discussed some of the aspects of both types of breast milk-related jaundice. For breast milk jaundice, many factors might be involved including the nutritional substances that are found within the human milk and the genetic factors which have been proven to play an essential role. On the other hand, breastfeeding jaundice is attributable to the lack of adequate human milk intake and/or inappropriate lactation habits. A careful diagnosis of the exact type of jaundice is essential to determine the management modality and prevent any potential exacerbation in case of misdiagnosis.

Funding: No funding sources

Conflict of interest: None declared

Ethical approval: Not required

\section{REFERENCES}

1. Ullah S, Rahman K, Hedayati M. Hyperbilirubinemia in Neonates: Types, Causes, Clinical Examinations, Preventive Measures and Treatments: A Narrative Review Article. Iran J Public Health. 2016;45(5):558-68.

2. Nag N, Halder S, Chaudhuri R, Adhikary S, Mazumder S. Role of bilirubin as antioxidant in neonatal jaundice and effect of ethanolic extract of sweet lime peel on experimentally induced jaundice in rat. Indian J Biochem Biophys. 2009;46(1):73-8.

3. Barikbin B, Yousefi M, Rahimi H, Hedayati M, Razavi SM, Lotfi S. Antioxidant status in patients with lichen planus. Clin Exp Dermatol. 2011;36(8):851-4.

4. Paludetto R, Mansi G, Raimondi F, Romano A, Crivaro V, Bussi $\mathrm{M}$, et al. Moderate hyperbilirubinemia induces a transient alteration of neonatal behavior. Pediatrics. 2002;110(4):50.

5. Nass RD, Frank Y. Cognitive and behavioral abnormalities of pediatric diseases. USA: Oxford University Press; 2010.

6. Preer GL, Philipp BL. Understanding and managing breast milk jaundice. Arch Dis Child Fetal Neonatal Ed. 2011;96(6):461-6.

7. Arias IM, Gartner LM, Seifter S, Furman M. Prolonged neonatal unconjugated hyperbilirubinemia associated with breast feeding and a steroid, pregnane-3(alpha), 20(beta)-diol, in maternal milk that inhibits glucuronide formation in vitro. J Clin Invest. 1964;43(11):2037-47.

8. Uhari M, Alkku A, Nikkari T, Timonen E. Neonatal jaundice and fatty acid composition of the maternal diet. Acta Paediatr Scand. 1985;74(6):867-73.

9. Lascari AD. 'Early' breast-feeding jaundice: clinical significance. J Pediatr. 1986;108(1):156-8. 
10. Auerbach KG, Gartner LM. Breastfeeding and human milk: their association with jaundice in the neonate. Clin Perinatol. 1987;14(1):89-107.

11. Soldi A, Tonetto P, Varalda A, Bertino E. Neonatal jaundice and human milk. J Matern Fetal Neonatal Med. 2011;24(1):85-7.

12. Kholy MS, Halim HY, Marzouk AH. Betaglucuronidase and hyperbilirubinemia in breast-fed versus formula-fed babies. J Egypt Public Health Assoc. 1992;67(3):237-48.

13. Maruo Y, Morioka Y, Fujito H, Nakahara S, Yanagi $\mathrm{T}$, Matsui K, et al. Bilirubin uridine diphosphateglucuronosyltransferase variation is a genetic basis of breast milk jaundice. J Pediatr. 2014;165(1):36-41.

14. Prameela KK. Breastfeeding during breast milk jaundice - a pathophysiological perspective. Med J Malaysia. 2019;74(6):527-33.

15. Chen S, Tukey RH. Humanized UGT1 Mice, Regulation of UGT1A1, and the Role of the Intestinal Tract in Neonatal Hyperbilirubinemia and Breast Milk-Induced Jaundice. Drug Metab Dispos. 2018;46(11):1745-55

16. Maisels MJ, Clune S, Coleman K, Gendelman B, Kendall A, McManus S, et al. The natural history of jaundice in predominantly breastfed infants. Pediatrics. 2014;134(2):340-5.

17. Bratton S, Cantu RM, Stern M. Breast Milk Jaundice. StatPearls Publishing. 2021.

18. Leung AK, Sauve RS. Breastfeeding and breast milk jaundice. J R Soc Health. 1989;109(6):213-7.

19. Poddighe D, Castelli L, Marseglia GL, Bruni P. Prolonged, but transient, elevation of liver and biliary function tests in a healthy infant affected with breast milk jaundice. BMJ Case Rep. 2014; 2014204124.

20. Management of hyperbilirubinemia in the newborn infant 35 or more weeks of gestation. Pediatrics. 2004;114(1):297-316.

21. Gartner LM. On the question of the relationship between breastfeeding and jaundice in the first 5 days of life. Semin Perinatol. 1994;18(6):502-9.

22. Schneider AP. Breast milk jaundice in the newborn. A real entity. JAMA. 1986;255(23):3270-4.

23. Section on Breastfeeding. Breastfeeding and the use of human milk. Pediatrics. 2012;129(3):827-41.

24. Calama MJ, Buñuel J, Valero MT, Labay M, Lasarte JJ, Valle F, et al. The effect of feeding glucose water to breastfeeding newborns on weight, body temperature, blood glucose, and breastfeeding duration. J Hum Lact. 1997;13(3):209-13.

25. Shrago L. Glucose Water Supplementation of the Breastfed Infant During the First Three Days of Life. J Human Lactation. 1987;3(3):82-6.
26. Goldberg NM, Adams E. Supplementary water for breast-fed babies in a hot and dry climate-not really a necessity. Arch Dis Child. 1983;58(1):73-4.

27. Righard L, Alade MO. Sucking technique and its effect on success of breastfeeding. Birth. 1992;19(4):185-9.

28. Weisman LE, Merenstein GB, Digirol M, Collins J, Frank G, Hudgins C. The effect of early meconium evacuation on early-onset hyperbilirubinemia. Am J Dis Child. 1983;137(7):666-8.

29. Gartner LM, Lee KS, Moscioni AD. Effect of milk feeding on intestinal bilirubin absorption in the rat. $\mathbf{J}$ Pediatr. 1983 Sep;103(3):464-71.

30. Stevenson DK, Bartoletti AL, Ostrander CR, Johnson JD. Pulmonary excretion of carbon monoxide in the human infant as an index of bilirubin production. IV. Effects of breast-feeding and caloric intake in the first postnatal week. Pediatrics. 1980;65(6):1170-2.

31. Maisels MJ, Gifford K. Normal serum bilirubin levels in the newborn and the effect of breast-feeding. Pediatrics. 1986;78(5):837-43.

32. Rubaltelli FF. Unconjugated and conjugated bilirubin pigments during perinatal development. IV. The influence of breast-feeding on neonatal hyperbilirubinemia. Biol Neonate. 1993;64(2-3):104-9.

33. Nielsen HE, Haase P, Blaabjerg J, Stryhn H, Hilden J. Risk factors and sib correlation in physiological neonatal jaundice. Acta Paediatr Scand. 1987;76(3):504-11.

34. Carvalho DM, Klaus MH, Merkatz RB. Frequency of breast-feeding and serum bilirubin concentration. Am J Dis Child. 1982;136(8):737-8.

35. Yamauchi Y, Yamanouchi I. The relationship between rooming-in/not rooming-in and breastfeeding variables. Acta Paediatr Scand. 1990;79(11):1017-22.

36. Kuhr M, Paneth N. Feeding practices and early neonatal jaundice. J Pediatr Gastroenterol Nutr. 1982;1(4):485-8.

37. Maisels MJ, Newman TB. Kernicterus in otherwise healthy, breast-fed term newborns. Pediatrics. 1995;96(4):730-3.

38. Gartner LM. Breastfeeding and jaundice. J Perinatol. 2001;21(S1):25-9.

Cite this article as: Shihab RAH, Samman AMB, Aqeel FA, Ghabrah ZT, Ghabrah OT, Ghannam AN, et al. Difference between breast milk jaundice and breast-feeding jaundice: literature review. Int $\mathbf{J}$ Community Med Public Health 2021;8:2559-63. 